\title{
Ge/ZnS-based micromachined Fabry-Perot filters for optical MEMS in the longwave infrared
}

\author{
Haifeng Mao, K. K. M. B. Dilusha Silva, Member, IEEE, Mariusz Martyniuk, Member, IEEE, \\ Jarek Antoszewski, John Bumgarner, John M. Dell, Member, IEEE and Lorenzo Faraone, Fellow, IEEE
}

\begin{abstract}
This paper reports on the successful demonstration of Ge/ZnS-based Fabry-Perot filters operating in the longwave infrared (LWIR). The suitability of thermally deposited Ge and $\mathrm{ZnS}$ as thin film mirror materials for micromachined LWIR Fabry-Perot filters has been fully investigated, and it is shown that a film growth temperature higher than $150{ }^{\circ} \mathrm{C}$ is key to depositing durable ZnS films. The optical constants of Ge and $\mathrm{ZnS}$ films in the LWIR band reveal that the material pair possesses high refractive index contrast and excellent LWIR transparency. Fixed-cavity LWIR Fabry-Perot filters with a 150- $\mu \mathrm{m}$ circular single-layer $\mathrm{Ge}$ top mirror and a four-layer $\mathrm{Ge} / \mathrm{ZnS} / \mathrm{Ge} / \mathrm{ZnS}$ bottom mirror were fabricated. Curvature in the suspended top mirror was corrected using a thin $\mathrm{SiN}_{\mathrm{x}}$ stress-compensation layer. After curvature correction, a mirror flatness of $550 \mathrm{~nm}$ was achieved, and the filter demonstrated a $60 \%$ peak transmission with a full-width at half-maximum of $700 \mathrm{~nm}$ as well as a outof-band rejection of $24: 1$.
\end{abstract}

Index Terms-Fabry-Perot, longwave infrared, LWIR, micromachining, multispectral, optical filter, thermal imaging.

\section{INTRODUCTION}

$\mathbf{L}$ ONGWAVE infrared (LWIR) multispectral imaging systems, capable of separating a completely passive thermal image into multiple spectral bands of interest within the 8-12 $\mu \mathrm{m}$ spectral range, not only enable better target identification but also can be used for material characterization and spectroscopy. As a field of increasing interest, LWIR multispectral imaging has been successfully deployed in various applications, including mineral mapping [1], [2], gas and volatile organic compound diagnostics [3]-[5], and water and land surface temperature monitoring [6], [7]. Conventional multispectral imagers employ mechanically-switched bulk filter systems to realize spectral selectivity. For example, based on the characteristic absorption of target analytes, Wabomba et al. [5] selected a set of LWIR bandpass filters with bandwidth of $0.5-1 \mu \mathrm{m}$, and integrated the filters in front of a detector array to produce wavelength-selective detection channels. This type of configuration, based on standard bulk optics, is typically characterized by significant size and cost, which prohibits their widespread use in many desirable applications. On the other hand, micro-electromechanical systems (MEMS) based Fabry-Perot tunable filters can be small and lightweight, and less costly to manufacture in comparison to their bulk filter counterparts.

The authors are with the School of Electrical, Electronics and Computer Engineering, University of Western Australia, Crawley, WA 6009, Australia (e-mail: 20878492@student.uwa.edu.au; dilusha.silva@uwa.edu.au; jarek.antoszewski@uwa.edu.au; john.dell@uwa.edu.au; lorenzo.faraone@uwa.edu.au).

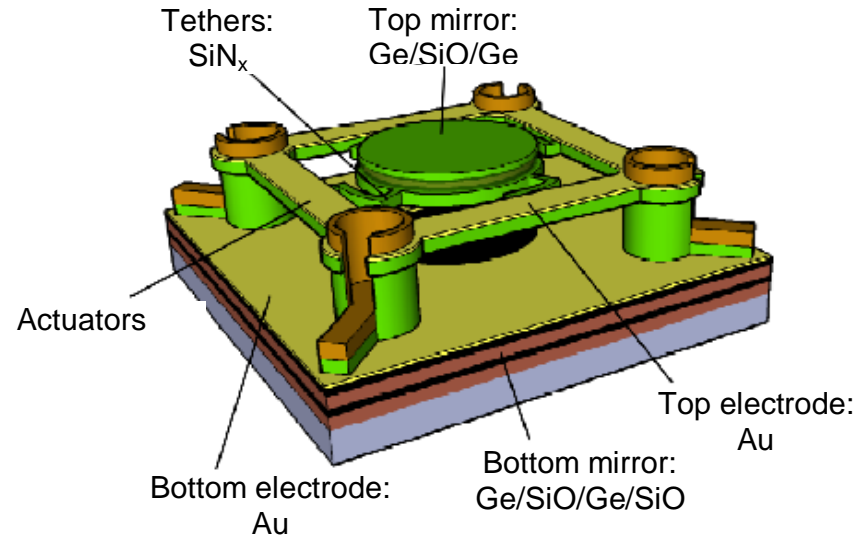

Fig. 1. 3D view of the MEMS-based shortwave infrared Fabry-Perot filter using Ge/SiO-based mirrors [13] developed by the Microelectronics Research Group at the University of Western Australia.

Whilst MEMS-based LWIR Fabry-Perot filters have been previously reported [8]-[12], the published works concentrate on achieving high spectral resolution by use of highly reflective multilayer Bragg mirrors. These reports include Teledyne [8] with $120 \mathrm{~nm}$ FWHM and InfraTec [9], [10] with $200 \mathrm{~nm}$ FWHM, using multilayer Ge/ZnS-based mirrors. Top mirror deformation, arising from stress mismatch between different mirror layers, complicates fabrication of these filters. Mirror curvature can degrade filter optical performance. Mirror stress gradients in these filters were balanced either by tailoring the layer stress conditions [8] or by using very thick, mechanically stiff carrier wafers [9], [10]. In comparison, VTT [11], [12] realized a filter with $110 \mathrm{~nm}$ FWHM based on Si/air/Si mirrors, which are not subject to film stress mismatch. However, this filter requires an undesirable high-temperature annealing process to control Si film stress to produce flat mirrors. The LWIR filter presented in this paper exploits a simple singlelayer top mirror approach to address the stress mismatch issue, and uses an additional stress-compensation layer to control residual top mirror stress gradient.

Our reseach team has previously demonstrated a MEMSbased shortwave infrared (SWIR: 1.6 to $2.5 \mu \mathrm{m}$ ) tunable FabryPerot filter for multispectral imaging applications [13]. The filter, as illustrated in Fig. 1, uses $\mathrm{SiN}_{\mathrm{x}}$ electrostatic beam actuators to move the suspended top mirror relative to a fixed bottom mirror, thus decreasing the mirror spacing and thereby allowing controllable tuning of the peak wavelength of the filter. Each mirror in the filter is a multilayer Bragg mirror structure composed of high-index-contrast and low-loss 
TABLE I

PERFormance ReQuirements of TunABle Filters For LWIR MultisPeCTRAL IMAGING APPLICATIONS

\begin{tabular}{ccc}
\hline \hline Parameter & Value & Comment \\
\hline Peak transmission & $>50 \%$ & Optical throughput for high SNR \\
\hline FWHM & $0.5-1 \mu \mathrm{m}$ & Wavelength discrimination \\
\hline Out-of-band rejection & $>10: 1$ & Wavelength discrimination \\
\hline
\end{tabular}

$\mathrm{Ge} / \mathrm{SiO}$ pairs. For transition of this technology into the LWIR band, although $\mathrm{Ge}$ is an ideal high-index material, $\mathrm{SiO}$ is far too absorbing [14]. There exists a wide variety of LWIR lowindex materials that could be considered as a replacement for the $\mathrm{SiO}$ layers, such as $\mathrm{ZnS}, \mathrm{ZnSe}$ and $\mathrm{BaF}_{2}$ [15]. Our choice of $\mathrm{ZnS}$ for the low-index medium to replace $\mathrm{SiO}$ has many advantages. Firstly, from a fabrication perspective, standard thermal evaporation can be used to deposit high quality thin films of both $\mathrm{Ge}$ and $\mathrm{ZnS}$, and both films exhibit good adhesion [16], [17]. Secondly, from an optical perspective, the material pair offers high refractive index contrast that can achieve highly reflective mirrors with a small number of periods. Moreover, because the refractive index of $\mathrm{ZnS}$ is close to the square root of that of the silicon substrate, a quarterwave $\mathrm{ZnS}$ layer can also serve as an adequate anti-reflection coating, thus negating the need of a third material.

In this paper, we report on the fabrication of durable Ge and $\mathrm{ZnS}$ thin films using thermal evaporation, and on a method to measure the optical constants of these films. Using Ge/ZnS as mirror materials, a fixed-cavity LWIR Fabry-Perot filter with single-layer top mirror has been designed and fabricated, demonstrating spectral characteristics that meet the optical requirements for LWIR multispectral imaging applications listed in Table I.

\section{THIN FILM FABRICATION}

Material durability of each thin film layer is a concern for both the filter fabrication process and the long-term stability and operation of the fabricated device. In order to successfully fabricate fully functional devices, the thin films need to withstand multiple exposures to both de-ionized (DI) water and the alkaline-based photoresist developer used in photolithography. High purity Ge (99.999 wt\% purity) and $\mathrm{ZnS}$ (99.99 wt\% purity) sources were used for thermal evaporation. The Ge and $\mathrm{ZnS}$ thin films were deposited by electron-beam evaporation and thermal evaporation, respectively. Whereas Ge films deposited at room temperature showed excellent durability under exposure to DI water and AZ400K developer, the ZnS films deposited at room temperature were found to delaminate immediately upon contact with these chemicals.

In this work, we have examined the effect of substrate heating during thin film deposition, on the adhesion of $\mathrm{ZnS}$ films to silicon and germanium. Silicon and germanium are the materials on which $\mathrm{ZnS}$ deposition takes place for bottom mirror formation (see section IV). Prior to deposition, the silicon and germanium substrates underwent a series of solvent washes followed by ultrasonic cleaning. ZnS layers of $1 \mu \mathrm{m}$ thickness were deposited by thermal evaporation at
TABLE II

DURABILITY of $1 \mu \mathrm{m}$ ThICK ZnS Films PREPARED AT DifFERENT SUBSTRATE DEPOSITION TEMPERATURES

\begin{tabular}{clc}
\hline \hline \multirow{2}{*}{ Substrate temperature $\left({ }^{\circ} \mathrm{C}\right)$} & \multicolumn{2}{l}{ The time taken to observe any delamination } \\
\cline { 2 - 3 } & In DI water & In AZ400K \\
\hline 50 & immediately & immediately \\
\hline 100 & 5 mins & 2 mins \\
\hline$\geq 150$ & $>2$ days & $>2$ days \\
\hline
\end{tabular}

a deposition rate of $0.2 \mathrm{~nm} / \mathrm{s}$ onto the substrates with the substrate temperature during deposition maintained at either $50{ }^{\circ} \mathrm{C}, 100{ }^{\circ} \mathrm{C}, 150{ }^{\circ} \mathrm{C}, 200{ }^{\circ} \mathrm{C}$ or $300^{\circ} \mathrm{C}$. To test the durability of these $\mathrm{ZnS}$ films, the samples were submerged in DI water and AZ400K developer, and the time taken to observe any evidence of delamination or cracking was recorded. The entire experimental procedure was repeated twice, and very similar results were obtained on both occasions. The results are shown in Table II.

As evident from Table II, the durability of $\mathrm{ZnS}$ films deposited at substrate temperatures higher than $150{ }^{\circ} \mathrm{C}$ is significantly improved in comparison to films deposited at room temperature. Such a dramatic improvement in the adhesion properties can be attributed to the higher surface mobility of adatoms during deposition at higher substrate temperatures, thereby improving the packing density and overall quality of the films [18], [19].

\section{Thin FILM OpticAl ChARACTERIZATION}

In order to characterize our deposition system and to establish that the deposited films have appropriate LWIR properties, the optical constants of the Ge films deposited at room temperature and the $\mathrm{ZnS}$ films deposited at $150{ }^{\circ} \mathrm{C}$ were studied. Although a variety of methods have been devised for determining the optical constants of thin films, such as ellipsometry [20] and film reflectance/transmittance measurements [21], we have chosen to investigate approaches that are compatible with our existing measurement capabilities, which rely on transmission spectra alone. Swanepoel et al. [22] reported on the use of fringe patterns of the transmission spectrum to calculate the optical constants of thin films; however, the primary technical challenge that prevents us from adopting this approach is the requirement to deposit sufficiently thick films to produce a sufficient number of fringes in the LWIR band. In this paper, we have implemented an optical transmission matrix model [23] based iterative analysis of the transmission spectrum to derive the optical constants.

The optical transmission matrix model can be explained as follows. Consider a multilayer thin film stack on a lossless substrate, where an individual layer $f$ has a thickness $d_{f}$, a refractive index $n_{f}$ and an extinction coefficient $k_{f}$, and the substrate has refractive index $n_{S}$. In the optical transmission matrix model, each layer is assigned a characteristic matrix, $M_{f}$, described as

$$
\left[M_{f}\right]=\left[\begin{array}{cc}
\cos \delta_{f} & \left(\mathrm{i} / \eta_{f}\right) \sin \delta_{f} \\
\mathrm{i} \eta_{f} \sin \delta_{f} & \cos \delta_{f}
\end{array}\right],
$$




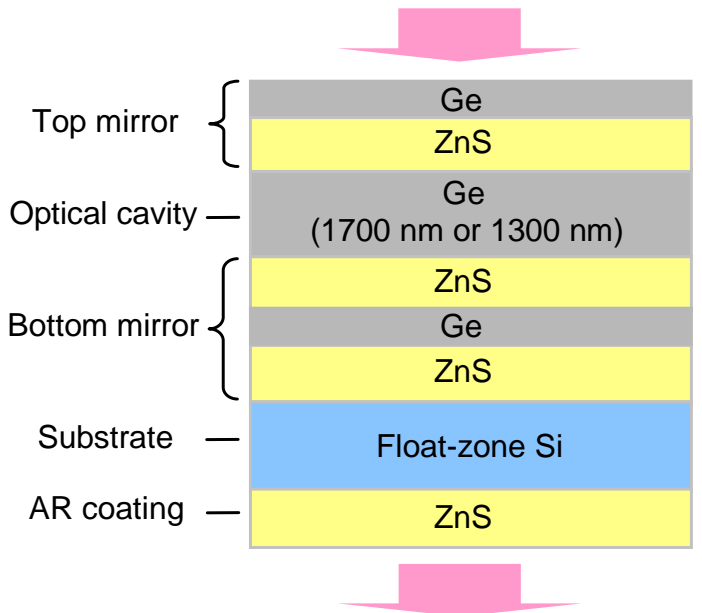

(a)

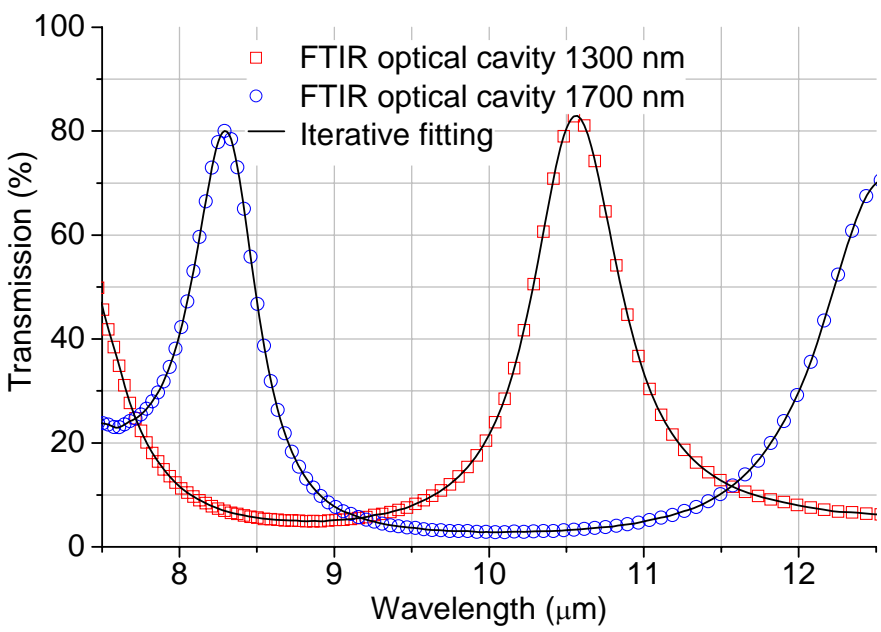

(b)

Fig. 2. (a) Layered structures used for determination of optical constants (b) Transmittance measured by FTIR (open data points) and the best fit to transmittance using an iterative procedure (solid lines).

where $\eta_{f}=\eta_{0}\left(n_{f}-\mathrm{i} k_{f}\right)$ and $\eta_{0}=2.65 \times 10^{-3} \Omega^{-1}$ are optical admittances of layer $f$ and free space, respectively, and $\delta_{f}=2 \pi d_{f}\left(n_{f}-\mathrm{i} k_{f}\right) / \lambda$ is the optical phase change in layer $f$. For light incident from air and propagating through $N$ thinfilm layers towards the substrate, the optical transmission $\mathrm{T}$ for the entire stack is calculated by first evaluating the quantities $\mathrm{B}$ and $\mathrm{C}$ as the matrix product,

$$
\left[\begin{array}{l}
B \\
C
\end{array}\right]=\left[M_{1}\right]\left[M_{2}\right] \cdots\left[M_{N}\right]\left[\begin{array}{c}
1 \\
\eta_{0} n_{S}
\end{array}\right],
$$

and then by

$$
T=\frac{4 \eta_{0}^{2} n_{S}}{\left(\eta_{0} B+C\right)\left(\eta_{0} B+C\right)^{*}}=\frac{4 \eta_{0}^{2} n_{S}}{\left|\eta_{0} B+C\right|^{2}} .
$$

Equation 3 can be used not only to calculate the optical transmittance of a multilayer stack given all the layer parameters, but also to extract unknown parameters of the constituent layers given a known stack transmittance.

Two multilayer structures, as depicted in Fig. 2a, consisting of multiple layers of $\mathrm{Ge} / \mathrm{ZnS}$ on the front side and a single layer of $\mathrm{ZnS}$ on the backside of silicon substrates, were fabricated for the purpose of determining the optical constants of the thin film layers. The substrates used in these structures were LWIR transparent float-zone (FZ) silicon wafers, because conventional Czochralski (CZ) silicon wafers exhibit strong LWIR absorption due to excessive oxygen content [24]. Apart from the middle Ge layer that was either $1700 \mathrm{~nm}$ or 1300 $\mathrm{nm}$ thick, which forms the optical cavity of the Fabry-Perot filter, all other Ge and $\mathrm{ZnS}$ layers that form the top and bottom mirrors were $600 \mathrm{~nm}$ and $1100 \mathrm{~nm}$ thick, respectively. Using the transmission spectra of these multilayer structures, rather than those of single layers, allows the optical constants of $\mathrm{Ge}$ and $\mathrm{ZnS}$ to be extracted simultaneously. In addition, the designed structures are essentially bandpass filters, which allows the optical performance to be evaluated at the same time.

Transmittance spectra for these structures were measured by Fourier transform infrared spectroscopy (FTIR), and are shown in Fig. 2b. The multilayer structure with a $1700 \mathrm{~nm}$ thick Ge layer as the optical cavity exhibited a second-order peak transmittance of $80 \%$ at the peak wavelength of $8.25 \mu \mathrm{m}$ and a fullwidth at half-maximum (FWHM) of $500 \mathrm{~nm}$. In comparison, for the structure with $1300 \mathrm{~nm}$ thick Ge layer as the optical cavity, the first-order peak transmittance and the FWHM were measured to be $85 \%$ at the peak wavelength of $10.5 \mu \mathrm{m}$ and $750 \mathrm{~nm}$, respectively. The measured high peak transmittance indicates that both the $\mathrm{Ge}$ and $\mathrm{ZnS}$ thin films are highly transparent in the LWIR. To derive thin film optical constants, a least-squares approach was implemented to fit the optical transmission matrix model (i.e. Equation 3) to the measured transmittance data of the two proposed multilayer structures. During the fit, refractive indices and extinction coefficients of Ge and ZnS thin film layers were iteratively varied, and layer thicknesses were fixed. Since both Ge and $\mathrm{ZnS}$ exhibit low dispersion in the LWIR band [25], [26], it was also assumed that the refractive indices and extinction coefficients of the investigated materials are constant over the entire LWIR range. When a goodness of fit of $99.9 \%$ was achieved, the optical constants of the electron-beam evaporated Ge and thermally evaporated $\mathrm{ZnS}$ were determined. Each multilayer structure determined one set of optical constants. The optical constants obtained from multiple similar multilayer structures were in excellent agreement. Table III compares these extracted optical constants with other published data.

\section{FABRY-PEROT Filter IMPLEMENTATION}

\section{A. Optical Design and Modelling}

The primary challenge for successful development of a Fabry-Perot LWIR filter is integration of the free-standing suspended top multilayer Bragg mirror. Due to the long wavelength being considered, a three-layer Ge/ZnS/Ge mirror stack is relatively thick (approximately $2.4 \mu \mathrm{m}$ ), and any stress mismatch between the constituting layers would highly deform the mirror and, thereby, severely degrade filter performance. In order to minimize any mirror bowing caused by stress gradients, the LWIR Fabry-Perot filter in this paper is designed to have a top suspended mirror comprised of a single quarterwavelength thick layer of Ge rather than a Ge/ZnS/Ge stack. 
TABLE III

Optical Constants of Ge and ZnS Thin Films at a WaVElength OF $10 \mu \mathrm{m}$

\begin{tabular}{cccc}
\hline \hline & \multicolumn{3}{c}{ Ge } \\
\cline { 2 - 4 } & This paper & Donovan [27] & Potter [25] \\
\hline$n$ & 4.0 & 3.98 & 4.0 \\
\hline$k$ & $2 \times 10^{-5}$ & 0 & 0 \\
\hline & \multicolumn{3}{c}{ ZnS } \\
\cline { 2 - 4 } & This paper & Hall [28] & Addamiano [26] \\
\hline$n$ & 2.2 & 2.17 & 2.2 \\
\hline$k$ & $2 \times 10^{-3}$ & $1 \times 10^{-3}$ & $8 \times 10^{-6}$ \\
\hline
\end{tabular}

The bottom mirror of the filter was a 4-layer Ge/ZnS/Ge/ZnS structure deposited on a FZ silicon substrate. The Ge and $\mathrm{ZnS}$ layers were designed to be of quarter-wave thickness at the design wavelength of $10 \mu \mathrm{m}$. Based on our experimental values of refractive indices ( $n=4.0$ for Ge and $n=2.2$ for $\mathrm{ZnS})$, the required thicknesses of the Ge and $\mathrm{ZnS}$ layers were determined to be $625 \mathrm{~nm}$ and $1136 \mathrm{~nm}$, respectively. Because the refractive index of thermally evaporated $\mathrm{ZnS}(n=2.2)$ is approximately the square root of that of the silicon substrate $(n=3.4)$, a quarter-wave $\mathrm{ZnS}$ layer was also deposited on the backside as an anti-reflection coating.

In the proposed asymmetric filter, since the top mirror has only a single quarter-wave optical layer and is less reflective than the bottom mirror, a relatively wide spectral bandwidth is expected because of the resulting lower cavity finesse. Calculated spectra with variable air cavity length, $d$, were modelled using Equation 3, and are shown in Fig. 3. The calculations indicate that the proposed filter is capable of achieving a spectral resolution of about $500 \mathrm{~nm}$ FWHM, and a peak transmittance of greater than $80 \%$ across the entire 8-12 $\mu \mathrm{m}$ LWIR band. The filter also features an out-ofband light transmission of $2 \%$. Given the peak transmission is above $80 \%$, the out-of-band rejection is therefore better than 40:1. These performance figures adequately fulfil the requirements of an optical filter for multispectral imaging applications introduced in Table I.

\section{B. Fabrication}

Prototype Fabry-Perot filters with a fixed air cavity gap were fabricated using surface micromachining. The filter structure and its cross-section are illustrated in Fig. 4a and Fig. 4b, respectively. First, a four-layer quarter-wave stack of $\mathrm{Ge} / \mathrm{ZnS} / \mathrm{Ge} / \mathrm{ZnS}$ for the bottom mirror was deposited on a FZ silicon wafer, followed another quarter-wave ZnS layer on the backside of the substrate to serve as an anti-reflection coating. A $200 \mathrm{~nm}$ thick Au layer was then deposited using thermal evaporation and lift-off, as an optical shield to block stray light from leaking around the filter onto the detector to be located optically behind the filter. The opening in this $\mathrm{Au}$ layer defines the optical aperture of the filter, and is $20 \mu \mathrm{m}$ laterally smaller than the mirror, to avoid unintended stray light due to misalignment errors. A sacrificial polyimide layer was then spin-coated on the sample, with a thickness of 5.6

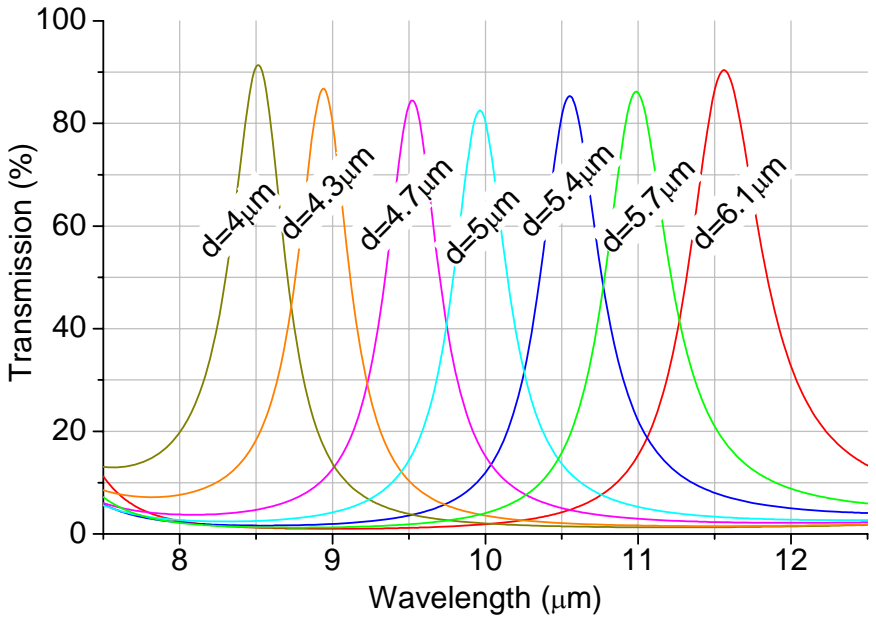

Fig. 3. The modelled spectra of the LWIR Fabry-Perot filter proposed in this paper, with variable cavity length $d$.

$\mu \mathrm{m}$. Patterning of the polyimide to create the four step-down support anchors to the substrate was accomplished by dry etching the polyimide in an $\mathrm{O}_{2}$ plasma with the use of a thick photoresist mask. A 5-nm thick compressively stressed ( $\sim 150 \mathrm{MPa}$ ) PECVD $\mathrm{SiN}_{\mathrm{x}}$ layer was then deposited and selectively removed in a $\mathrm{CF}_{4}$ plasma from everywhere except directly below the top mirror. This $\mathrm{SiN}_{\mathrm{x}}$ layer plays a role in balancing the compressive stress gradients inherent in the top Ge mirror, thereby reducing mirror bowing. The $\mathrm{SiN}_{\mathrm{x}}$ layer, at several nanometres thick, has a negligible effect on the optical performance of the filter. A 250-nm thick Ge layer was then deposited using lift-off to form part of the top mirror, the tethers and beams, and the structural support for the suspended top mirror. Another 375-nm Ge layer was lift-off deposited in the mirror area, which, combined with the previous 250 $\mathrm{nm}$ Ge layer, forms a complete quarter-wave top mirror. An array of $4 \mu \mathrm{m}$ diameter holes were etched to perforate the top mirror using a $\mathrm{CF}_{4}$ plasma in order to facilitate the structural release process via removal of the sacrificial polyimide layer in an $\mathrm{O}_{2}$ plasma. These holes allow the mirror to be fully released within a relatively short time and, therefore, prevent cracks forming in the Ge top mirror due to overheating from over-exposure to the $\mathrm{O}_{2}$ release plasma. Importantly, stray light transmitted through the etch holes was shielded by an array of circular Au micropatterns, deposited on the bottom mirror directly below the etch hole array. A scanning electron microscope (SEM) image giving the structural dimensions of the fabricated device is shown in Fig. 4c.

\section{Filter Characterization}

Mirror flatness characteristics of the fabricated filters have been studied using a noncontact optical profilometer. Fig. 5 shows line scans of the top mirror surface profiles taken from profilometry measurements for two fabricated filters, with and without a $\mathrm{SiN}_{\mathrm{x}}$ stress-compensation layer. It is revealed that the top mirror of the filter without the $\mathrm{SiN}_{\mathrm{x}}$ stresscompensation layer exhibited a large convex center-to-edge bowing of $1.5 \mu \mathrm{m}$. This bowing is caused by the relief of 


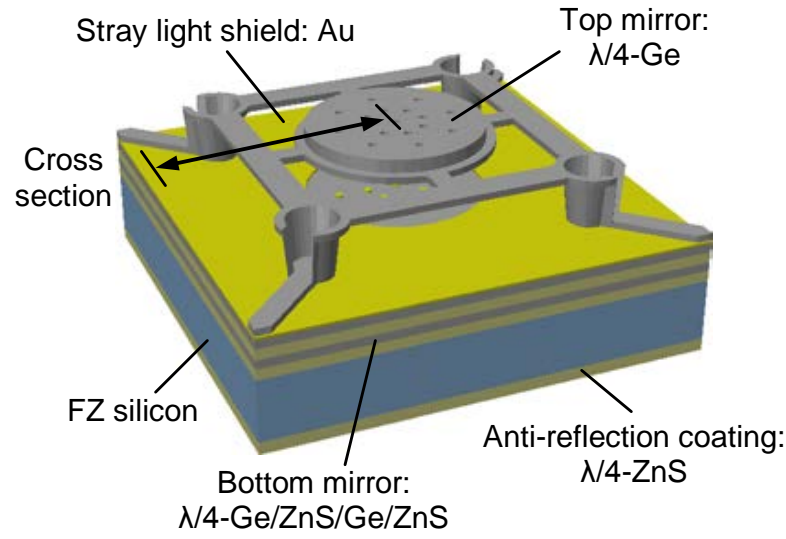

(a)

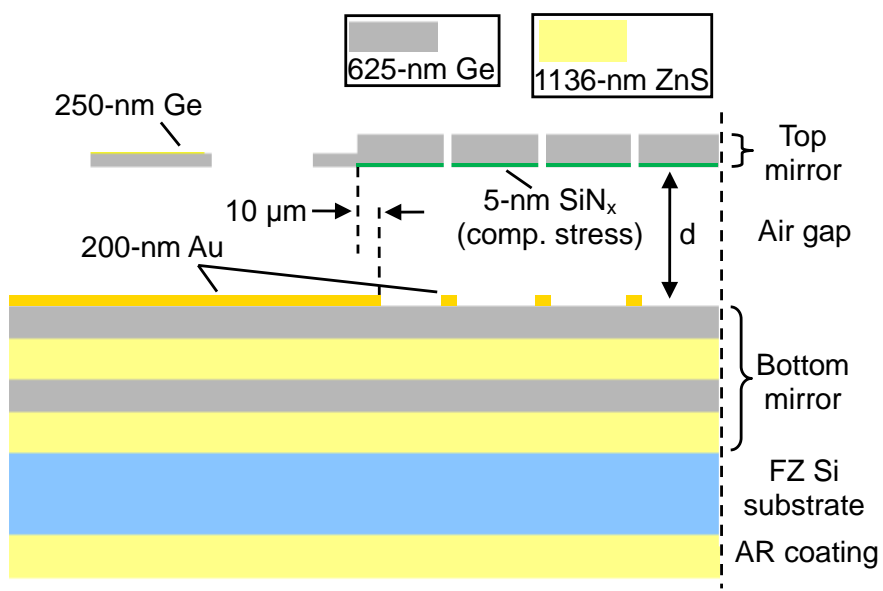

(b)

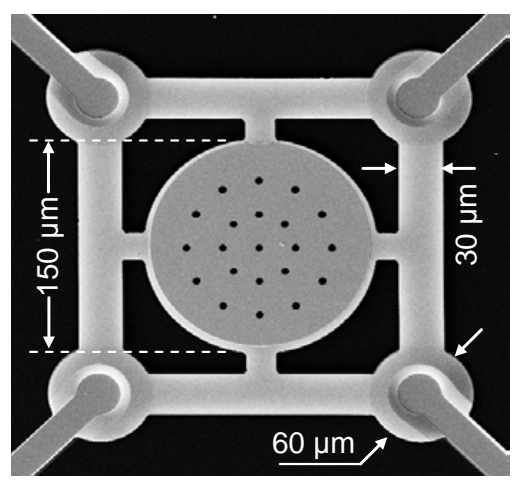

(c)

Fig. 4. (a) Schematic view of the LWIR Fabry-Perot filter proposed in this paper, and (b) cross section view of the filter, and (c) top view scanning electron micrograph of the fabricated LWIR Fabry-Perot filter and its structural dimensions.

residual in-built compressive stress gradients in the suspended structure. By the use of a $\mathrm{SiN}_{\mathrm{x}}$ stress-compensation layer, the mirror bowing became significantly less convex, with the center-to-edge amplitude reduced to $550 \mathrm{~nm}$. It is also noted that the fabricated mirrors exhibited no noticeable tilt.

The optical transmittance of the fabricated Fabry-Perot filters was measured as a function of wavelength using FTIR, and is presented in Fig. 6. The optical model curves shown in Fig. 6 include the effects of mirror bowing as measured by profilometry. Table IV compares the measured spectral characteristics

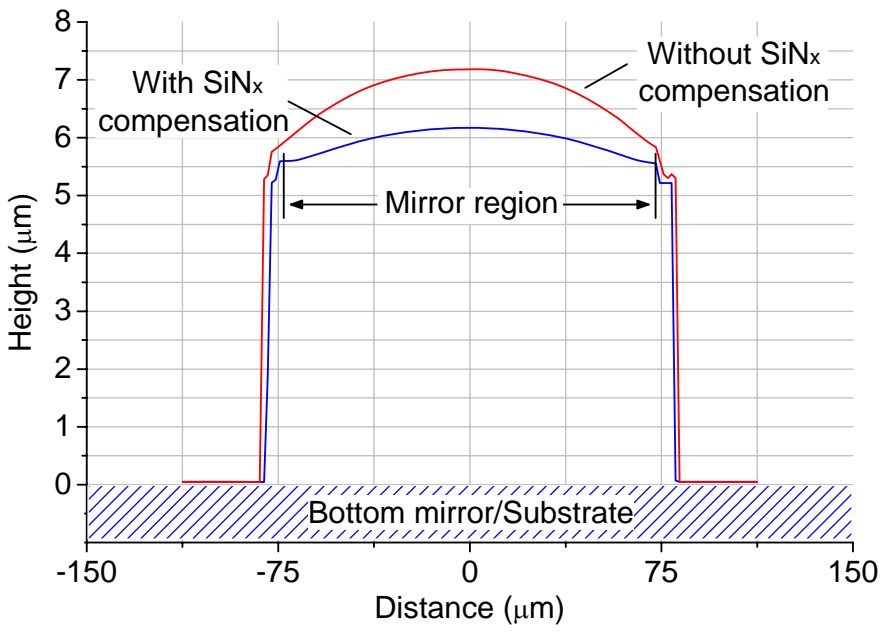

Fig. 5. Measured top mirror surface profiles of the fabricated LWIR FabryPerot filters, using an optical profilometer.

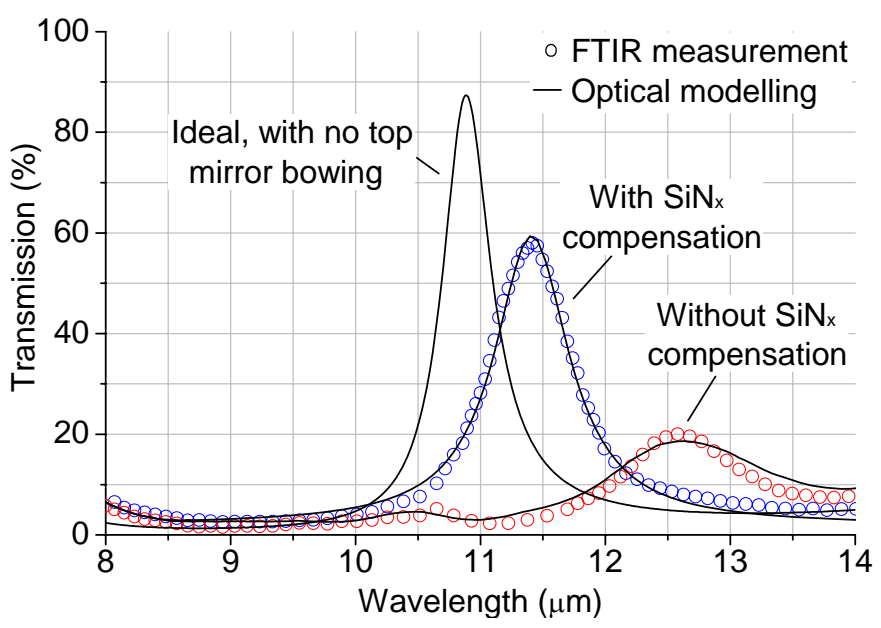

Fig. 6. The measured transmission spectra (open data points) and the modelled transmission spectra (solid line) of the fabricated LWIR Fabry-Perot filters. The modelling includes the effects due to top mirror bowing.

with the design values, as well as with the optical requirements of LWIR filters for multispectral imaging applications. It can be clearly seen that transmission characteristics of the filter in the absence of $\mathrm{SiN}_{\mathrm{x}}$ stress-compensation layer are severely degraded due to the large mirror bowing, showing a poor peak transmittance of $20 \%$, a wide FWHM of $1.3 \mu \mathrm{m}$ and a small out-of-band rejection of $8: 1$; all of which are far from fulfillment of the optical requirements for LWIR multispectral imaging applications. In comparison, the transmission curve of the filter with a $\mathrm{SiN}_{\mathrm{x}}$ stress-compensation layer shifts to shorter wavelengths, exhibiting a higher peak transmittance of $60 \%$, a narrower FWHM of $700 \mathrm{~nm}$ and a larger out-ofband rejection ratio of $24: 1$. Such a dramatic improvement in the transmission characteristics and the observed blueshift in the peak transmission wavelength can be attributed to the mirror bowing reduction and the accompanying decrease in mirror separation, respectively, as indicated in Fig. 5. It is evident that despite still showing some degree of performance degradation in comparison to the intended design parameters, 
TABLE IV

COMPARISON BETWEEN THE ACHIEVED SPECTRAL CHARACTERISTICS, The Designed VAlues, AND THE VAlues RequiRed For FABRy-Perot FILTERS IN LWIR MULTISPECTRAL IMAGING APPLICATIONS.

\begin{tabular}{ccccc}
\hline \hline \multirow{2}{*}{ Parameter } & \multirow{2}{*}{ Required } & \multirow{2}{*}{ Design } & \multicolumn{2}{c}{ Achieved } \\
\cline { 3 - 5 } & & & w/o SiN $\mathrm{x}$ & w/ $\mathrm{SiN}_{\mathrm{x}}$ \\
\hline Peak transmission & $>50 \%$ & $>80 \%$ & $20 \%$ & $60 \%$ \\
\hline Spectral width & $0.5-1 \mu \mathrm{m}$ & $500 \mathrm{~nm}$ & $1.3 \mu \mathrm{m}$ & $700 \mathrm{~nm}$ \\
\hline Out-of-band rejection & $>10: 1$ & $>40: 1$ & $8: 1$ & $24: 1$ \\
\hline
\end{tabular}

the fabricated filter prototype that employs a $\mathrm{SiN}_{\mathrm{x}}$ stresscompensation layer sufficiently meets the optical requirements for LWIR multispectral imaging applications, provided that it is incorporated with an appropriate electrostatic actuation mechanism.

\section{CONCLUSIONS}

A micromachined LWIR tunable optical filter is essential for the future realization of low-cost and robust LWIR multispectral imaging systems. In this paper, we have proposed a LWIR filter technology that is based on a MEMS Fabry-Perot optical cavity that has previously been demonstrated in the shortwave infrared region [13]. The direct extension of this technology to LWIR wavelengths is problematic, because the SWIR low index mirror material $\mathrm{SiO}$ is excessively absorbing in the LWIR. This paper has presented the preparation and LWIR characterization of electron-beam deposited Ge and thermally deposited $\mathrm{ZnS}$ thin films for mirror formation, as well as implementation of these components to realize a micromachined LWIR Fabry-Perot filter. It was shown that while durable Ge films could be deposited at room temperature, high quality $\mathrm{ZnS}$ films need to be deposited at substrate temperatures above $150{ }^{\circ} \mathrm{C}$. An iterative least-squares approach was successfully applied to extract the optical constants of Ge and $\mathrm{ZnS}$ thin films in the LWIR band. The refractive indices of Ge and $\mathrm{ZnS}$ were determined to be 4.0 and 2.2, respectively, showing high index contrast. The extinction coefficients of Ge and $\mathrm{ZnS}$ were determined to be $2 \times 10^{-5}$ and $2 \times 10^{-3}$, respectively, showing excellent optical transparency. The Ge/ZnS material system was then considered in a LWIR Fabry-Perot filter design. While the filter was predicted to have at least $80 \%$ peak transmittance, a FWHM spectral width of approximately 500 $\mathrm{nm}$, and an out-of-band rejection better than 40: 1 across the entire $8-12 \mu \mathrm{m}$ range, the fabricated prototype filter with the employment of a mirror-stress compensation layer achieved a peak transmittance of $60 \%$, a bandwidth of $700 \mathrm{~nm}$, and an out-of-band rejection of 24: 1. Bowing of the suspended top mirror due to insufficient compensation of stress gradients has been identified as the primary source of degradation of optical performance. Although the measured filter performance characteristics did not achieve the theoretically predicted performance, they adequately satisfy the system requirements for multispectral LWIR imaging. Overall, the results presented in this paper represent an excellent starting point for the realization of tunable LWIR Fabry-Perot filters which can be integrated optically ahead of a detector array to realize
LWIR multispectral imaging systems. However, it should be noted that these results only showed partial correction of mirror bowing, and peak performance has not yet been demonstrated. This result also implies that the specifications for LWIR multispectral imaging scenarios (Table I) will not so easily be achieved for larger diameter filters because of excessive mirror distortion. In order to fabricate filters that suit a wider application scope, a more compressive SiNx stresscompensation layer, which allows for complete balance of mirror stress, is needed.

\section{REFERENCES}

[1] A. B. Kahle and A. F. H. Goetz, "Mineralogic information from a new airborne thermal infrared multispectral scanner," Science, vol. 222, no. 4619, pp. 24-27, Oct. 1983.

[2] R. G. Vaughan, S. J. Hook, W. M. Calvin, and J. V. Taranik, "Surface mineral mapping at Steamboat Springs, Nevada, USA, with multi-wavelength thermal infrared images," Remote Sens. Environ., vol. 99, no. 1-2, pp. 140-158, Nov. 2005

[3] L. Zhang and G. W. Small, "Automated detection of chemical vapors by pattern recognition analysis of passive multispectral infrared remote sensing imaging data," Appl. Spectrosc., vol. 56, no. 8, pp. 1082-1093, Feb. 2002.

[4] S. Teggi, M. P. Bogliolo, M. F. Buongiorno, S. Pugnaghi, and A. Sterni, "Evaluation of $\mathrm{SiO}_{2}$ emission from Mount Etna using diurnal and nocturnal multispectral IR and visible imaging spectrometer thermal IR remote sensing images and radiative transfer models," J. Geophys. Res.: Sol. Ea., vol. 104, no. B9, pp. 20069-20079, Sep. 1999.

[5] M. J. Wabomba, Y. Sulub, and G. W. Small, "Remote detection of volatile organic compounds by passive multispectral infrared imaging measurements," Appl. Spectrosc., vol. 61, no. 4, pp. 349-358, Apr. 2007.

[6] W. B. Clodius, C. Borel, L. Balick, and S. J. Hook, "Validation of the MTI water surface temperature retrieval algorithms," in Proc. IEEE Int. Geosci. Remote Sens. Symp. (IGARSS'02), 2002, pp. 30-32.

[7] A. P. Rodger, L. K. Balick, and W. B. Clodius, "The performance of the multispectral thermal imager (MTI) surface temperature retrieval algorithm at three sites," IEEE Trans. Geosci. Remote Sens., vol. 43, no. 3, pp. 658-665, Mar. 2005.

[8] P. A. Stupar, R. L. Borwick, J. F. DeNatale, P. H. Kobrin, and W. J. Gunning, "MEMS tunable Fabry-Perot filters with thick, two sided optical coatings," in Proc. IEEE 15th Int. Conf. Solid-State Sens., Actuators, Microsyst. (TRANSDUCERS'09), Denver, CO, 2009, pp. 1357-1360.

[9] M. Ebermann, M. Meinig, S. Kurth, K. Hiller, E. Gittler, and N. Neumann, "Tiny mid- and long-wave infrared spectrometer module with a MEMS dual-band Fabry-Perot filter," in Proc. Sensor+Test Conf.: IRS ${ }^{2}$, Nürnberg, 2011, pp. 94-99.

[10] M. Meinig, M. Ebermann, N. Neumann, S. Kurth, K. Hiller, and T. Gessner, "Dual-band MEMS Fabry-Perot filter with two movable reflectors for mid- and long-wave infrared microspectrometers," in Proc. IEEE 16th Int. Conf. Solid-State Sens., Actuators, Microsyst. (TRANSDUCERS'11), Beijing, China, 2011, pp. 2538-2541.

[11] M. Tuohiniemi, A. Nasila, and J. Makynen, "Characterization of the tuning performance of a micro-machined Fabry-Perot interferometer for thermal infrared," J. Micromech. Microeng., vol. 23, no. 7, pp. 1-7, Jul. 2013.

[12] M. Tuohiniemi, M. Blomberg, A. Akujarvi, J. Antila, and H. Saari, "Optical transmission performance of a surface-micromachined FabryPerot interferometer for thermal infrared," J. Micromech. Microeng., vol. 22, no. 11, pp. 1-7, Sep. 2012.

[13] J. S. Milne, J. M. Dell, A. J. Keating, and L. Faraone, "Widely tunable MEMS-based Fabry-Perot filter,” J. Microelectromech. Syst., vol. 18, no. 4, pp. 905-913, Jul. 2009.

[14] H. R. Philipp, "Silicon monoxide (SiO) (noncrystalline)," in Handbook of Optical Constants of Solids, vol. 1, E. D. Palik, Ed. Burlington: Elsevier, 2012, ch. 36, p. 769.

[15] Janos Technology LLC. Optical Materials Guide. [online]. Available: http://www.janostech.com/knowledge-center/optical-materialsguide.html, accessed Apr. 29, 2015.

[16] D. Ban, F. Yang, R. Fang, S. Xu, and P. Xu, "Interface formation of $\mathrm{Ge} / \mathrm{ZnSe}(100)$ and $\mathrm{Ge} / \mathrm{ZnS}(111)$ heterojunctions studied by synchrotron radiation photoemission," Acta Phys. Sin., vol. 5, no. 8, pp. 590-600, Aug. 1996 
[17] A. D. Sio, A. Giannini, G. Dionisio, L. Gambicorti, P. Bianchi, M. Ciofini, L. Mercatelli, and E. Pace, "Development of an infrared wide pass band germanium filter," Dep. Astro. Space Sci., Firenze Univ., Italy, Tech. Rep. TR01-2008, Mar. 2008.

[18] M. Zhou, D. Liu, T. Yu, and Q. Cai, "The optical properties of different temperature deposited $\mathrm{ZnS}$ film in visible to near-infrared region," in Proc. SPIE 6th Int. Symp. on Advanced Optical Manufacturing and Testing Technologies, Xiamen, China, Oct. 2012, pp. 84192-84195.

[19] M. Ashraf, S. M. J. Akhtar, Z. Ali, and A. Qayyum, "The influence of substrate temperature on the structural and optical properties of $\mathrm{ZnS}$ thin films," Semiconductors, vol. 45, no. 5, pp. 699-702, May. 2011.

[20] H. C. Ong and R. P. H. Chang, "Optical constants of wurtzite ZnS thin films determined by spectroscopic ellipsometry," Appl. Phys. Lett., vol. 79, no. 22, pp. 3612-3614, Nov. 2001.

[21] E. Khawaja and S. G. Tomlin, "The optical constants of thin evaporated films of cadmium and zinc sulphides," J. Phys. D: Appl. Phys., vol. 8, no. 5, pp. 581-594, Apr. 1975.

[22] R. Swanepoel, "Determination of the thickness and optical constants of amorphous silicon," J. Phys. E: Sci. Instrum., vol. 16, no. 12, pp. 12141222, Dec. 1983.

[23] H. A. Macleod, "Basic theory," in Thin-film Optical Filters, 3rd ed. Bristol: Institute of Physics Publishing, 2001, ch. 2, pp. 43-45.

[24] W. S. Lau, "The measurement of oxygen and carbon and other impurities in silicon," in Infrared Characterization for Microelectronics. Singapore: World Scientific, 1999, ch. 3, pp. 25-30.

[25] R. F. Potter, "Germanium (Ge)," in Handbook of Optical Constants of Solids, vol. 1, E. D. Palik, Ed. Burlington: Elsevier, 2012, ch. 17, pp. 475-476.

[26] E. D. Palik and A. Addamiano, "Zinc sulfide (ZnS)," in Handbook of Optical Constants of Solids, vol. 1, E. D. Palik, Ed. Burlington: Elsevier, 2012, ch. 27, pp. 613-614.

[27] T. M. Donovan, W. E. Spicer, J. M. Bennett, and E. J. Ashley, "Optical properties of amorphous germanium films," Phys. Rev. B, vol. 2, no. 2, pp. 397-413, Jul. 1970.

[28] J. F. Hall, JR., and W. F. C. Ferguson, "Optical properties of cadmium sulfide and zinc sulfide from 0.6 micron to 14 microns," J. Opt. Soc. Am., vol. 45, no. 9, pp. 714-717, Sep. 1955.

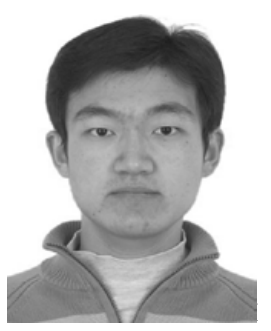

Haifeng Mao was born in China in 1985. He received the B.Eng. degree in Physics from Dalian University of Technology, China, in 2007, and M.Sc. degree in Sensor Systems Technology from Karlsruhe University of Applied Sciences, Germany, in 2010. He is currently pursuing the Ph.D. degree at the School of Electrical, Electronic and Computer Engineering, University of Western Australia, Perth, Australia. His current research activities involve design, fabrication and characterization of optical MEMS filters for multispectral imaging applications.

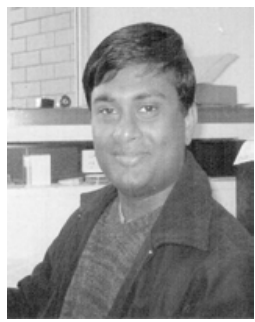

K. K. M. B. Dilusha Silva was born in Sri Lanka in 1973. He received the Honours degrees in Physics and Electronic Engineering from the University of Western Australia (UWA), and the Ph.D. degree in optical imaging technologies for biomedical applications in 2004

He has worked both in industry and academia, and is currently a Research Professor and the Engineering Manager with the Microelectronics Research Group, UWA. Since returning to UWA in 2009, his research interests include optical MEMS sensors, optical spectroscopic sensors, and MEMS biosensors. He has attracted funding for his research from the agriculture and aerospace sectors, and the government, and is presently leading a number of MEMS related research efforts at MRG, with strong commercial links to both the agricultural and aerospace sectors.

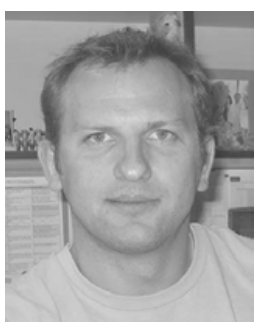

Mariusz Martyniuk was born in Poland in 1976. $\mathrm{He}$ received the B.Sc. (Hons.) degree from the University of Toronto, ON, Canada, the M.A.Sc. degree from McMaster University, ON, Canada, and the Ph.D. degree from the University of Western Australia, Perth, Australia, in 2007.

$\mathrm{He}$ worked in the industry sector as an Electronics Engineer before rejoining UWA, where he is currently a Research Professor with the Microelectronics Research Group and manages the Western Australian Node of the Australian National Fabrication Facility. His primary areas of interest encompass thin-film materials and thin-film mechanics, as well as their applications in micro-electromechanical systems and optoelectronic devices.

Dr. Martyniuks research contributions were recognized by the award of the Inaugural Australian Museum Eureka Prize (the Oscars of Australian science) for Outstanding Science in Support of Defence or National Security in 2008.

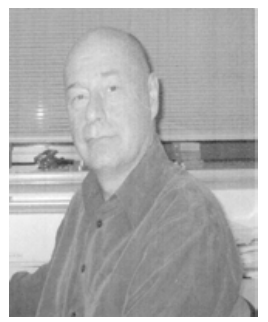

Jarek Antoszewski received the Master degree in Physics from Teachers College in Olsztyn, Olsztyn, Poland, in 1977, and the Ph.D. degree in semiconductor physics from the Institute of Physics of Polish Academy of Science, Warsaw, Poland, in 1982.

From 1982 to 1990, he was a Researcher and then a Project Leader with the Research and Development Laboratory, WILMER Ltd., Warsaw, where he was involved in design and testing of infrared radiometers for industrial applications. In 1991, he joined the Microelectronics Research Group, University of Western Australia, Perth, WA, Australia, where he has been engaged in magneto-transport studies of semiconductor materials, and physics and technology of infrared detectors. His current research interests include the development of technology merging II-VI and III-V based infrared detectors with microelectromechanical systems (MEMS) technology into a new technology for monolithic and tunable infrared sensors.

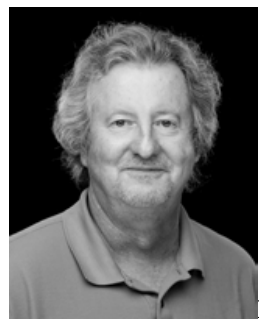

John Bumgarner received the B.S. and M.S. degrees in materials science and engineering from Northwestern University, and the Ph.D. degree in materials science and engineering from North Carolina State University with a focus on wide band gap materials, $\mathrm{SiC}$, and CVD of dielectrics in 1995.

He was a Process Integration Engineer and the Manager with Intel Corporation, a Laboratory Director with SRI International, and the Operations Director with Stanford University NanoFab. He is currently an Associate Research Professor with the University of Western Australia. His current interests are in research and development of optical microelectromechanical systems devices.

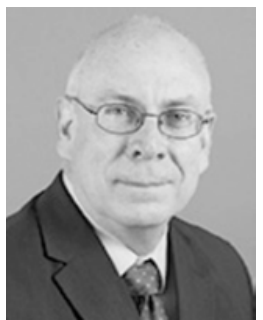

John M. Dell is currently a Professor of Electrical Engineering with the University of Western Australia, Perth, WA, Australia.

His primary areas of interest are semiconductor optoelectronics and optical microelectromechanical systems (MEMS) devices. He has worked in both industry and academia in these fields. Work undertaken by his group on robust and low-cost microspectrometer technology has attracted funding from the US and the Australian Department of Defence, and more recently from the Australian Grains Research and Development Corporation. This latter funding is for the development of low-cost tools using infrared spectroscopy for broad acre agriculture applications. This work is being undertaken as a collaboration between Electrical Engineers and Soil Scientists. He has several patents, and over 200 journal and conference publications 


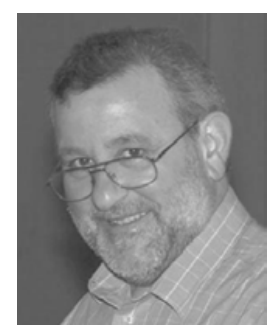

Lorenzo Faraone was born in Italy in 1951. He received the Ph.D. degree from the University of Western Australia (UWA), Perth, WA, Australia, in 1979.

He was a Research Scientist with Lehigh University, Bethlehem, PA, USA, from 1979 to 1980 , where he was involved in studies on MOS devices. From 1980 to 1986 , he was a Member of the Technical Staff with RCA Laboratories, David Sarnoff Research Center, Princeton, NJ, USA, where he was involved in very large scale integration (VLSI) CMOS and non-volatile memory technologies, and space radiation effects in siliconon-sapphire MOS integrated circuits. He joined the School of Electrical, Electronic and Computer Engineering, UWA, in 1987, where he has been a Professor since 1998, and the Head of the Department/School from 1999 to 2003. Since joining UWA, his research interests have been in the areas of compound semiconductor materials and devices, and microelectromechanical systems (MEMS). He has supervised more than $30 \mathrm{Ph}$.D. student completions, and published more than 300 refereed technical papers in journals and conference proceedings.

Prof. Faraone was awarded the RCA Laboratories Individual Outstanding Achievement Award in 1983 and 1986, and the John de Laeter Innovation Award in 1997. He is a Member of the Order of Australia (AM), and a Fellow of both the Australian Academy of Science (FAA) and the Australian Academy of Technological Sciences and Engineering (FTSE). 